\title{
The X-15 3-65 Accident: An Aircraft Systems and Flight Control Perspective Jeb S. Orr ${ }^{(1)}$, Irving C. Statler ${ }^{(2)}$, and Immanuel Barshi ${ }^{(3)}$
}

\begin{abstract}
Despite the NASA X-15 program's outstanding success in developing and operating the first manned hypersonic research platform, the program suffered a fatal accident on November 15, 1967, when X-15-3, the only aircraft outfitted with advanced pilot displays and an adaptive flight control system, was lost after entering uncontrolled flight at an altitude of 230,000 feet and a velocity near Mach 5. The pilot, Major Michael J. Adams, was incapacitated by the aircraft accelerations and was killed either during the ensuing breakup or upon ground impact.
\end{abstract}

In light of mitigating risk to current and emerging manned aerospace vehicles, a comprehensive systemslevel analysis of the accident is presented with a focus on the electrical power, flight control, and instrumentation failures that affected not only the vehicle dynamics but substantially impacted the pilot decisions that led to an inevitable loss of control. Insights based on reconstructed flight data as well as analysis and simulation of the $X-15$ 's unique adaptive control system, yield new conclusions about the reasons for the control system's anomalous behavior and the system-level interactions and human-machine interface design oversights that led to the accident.

\subsection{INTRODUCTION}

The X-15 research airplane (Figure 1.0-1) is widely regarded as one of the most successful highperformance flight research platforms ever developed with its 199 flights and nearly 10-year operational history [1]. Many advances in the modern understanding of hypersonic flight mechanics, including thermal protection, structural design, shockimpingement heating, air-data sensing, and hypersonic propulsion can be directly attributed to research performed using the X-15 platform.

${ }^{(1)}$ Jeb S. Orr, The Charles Stark Draper Laboratory, Inc., 1500 Perimeter Parkway, Ste 225, Huntsville, AL 35806; jeb.orr@nasa.gov

${ }^{(2)}$ Irving C. Statler, NASA Ames Associate, 1362

Cuernavaca Circulo, Mountain View, CA 94040; icstatler@earthlink.net

(3) Immanuel Barshi, M/S 262-4; NASA Ames Research Center, CA 94035-1000; immanuel.barshi@nasa.gov
The X-15 program conducted 199 research flights over a period of nearly 10 years. During this period, the X15 set altitude and speed records for a piloted aerospace plane, and ultimately held the record for the highest altitude $(354,200$ feet; August 22, 1963) and highest Mach number (Mach 6.7; October 3, 1967) for a winged vehicle until the atmospheric reentry of Space Shuttle STS-1 in 1981.

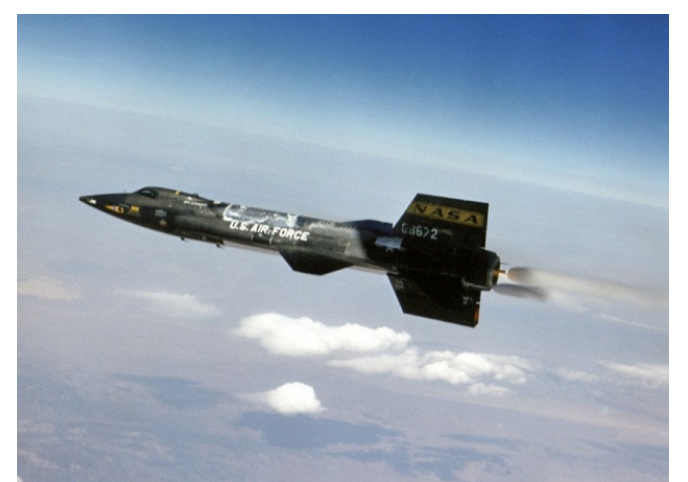

Figure 1.0-1. X-15-3 in Flight (USAF photo)

On November 15, 1967, X-15-3 was destroyed during the 65 th flight of $X-15-3$ and the 191 st flight of the $\mathrm{X}-15$ program as a result of a structural load exceedance precipitated by a loss of control [2]. The research pilot, United States Air Force (USAF) Major Michael J. Adams, was killed.

The causes of the accident are complex, beginning with an electrical anomaly associated with a drive motor of experimental equipment that had not been properly qualified for the space environment. This electrical anomaly led to a series of electrical and instrumentation failures that will be discussed in detail in the following sections.

A concern has recently arisen within the aerospace community as to the implications of the X-15-3 accident as to potential requirements for flight control design, vehicle instrumentation, crew spatial/situational awareness, and abort modes of emerging manned hypersonic vehicles, launch vehicles, and spacecraft. Furthermore, some aspects of the anomalies experienced on Flight 3-65 have been incorrectly reported in the literature. Until recently, the accident has not been treated at a comprehensive systems level considering the human factors, flight controls, and subsystem anomalies simultaneously.

The January 1968 report of the joint NASA/USAF Accident Investigating Board [2] that reviewed the 
causes of the destruction of the $\mathrm{X}-15-3$ is a primary source for the present discussion. Importantly, the report lacks detail especially with respect to the function of the MH-96 adaptive flight control system (AFCS). After an extensive search, many references were identified that provide additional insight into the operation of various aircraft subsystems. The present report is of a study undertaken to assemble a complete picture of the aircraft and its systems at the time of the accident.

The failure analysis of Flight 3-65 has also been considered from a perspective of the human factors of the accident. The design of the pilot interfaces with the AFCS and of the instrument displays, the interaction of the pilot both with the aircraft systems and with ground control, and the management of the program at the time of the accident were important aspects of the event and are addressed in detail in a companion paper [3].

\subsection{THE X-15 RESEARCH AIRCRAFT}

The X-15 was a piloted, single-seat rocket-propelled hypersonic research airplane developed under cooperative funding and program management from the USAF, NASA, and the United States Navy (USN). Initial design concept engineering was completed in the early 1950's and a prime contract for three aircraft was awarded to North American Aviation in September 1955. The first of three airframes was completed in October of 1958.

An internal layout drawing of the $\mathrm{X}-15$ airplane is shown below in Figure 2.0-1. The X-15 cockpit consisted of a single seat just aft of the RCS compartment and forward of the equipment bay. The cockpit used a relatively close-fitting canopy having two planar windows. Aft of the pilot station was an equipment bay containing most of the research instrumentation.

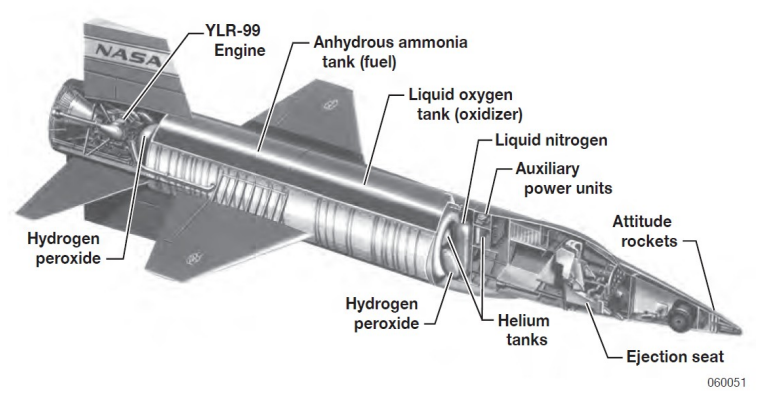

Figure 2.0-1. X-15 Research Airplane Internal Layout (NASA)

The X-15 was carried aloft to a launch altitude of 45,000 feet by a NASA NB-52 aircraft and the flight typically lasted 10 minutes from launch to touchdown [1]. The X-15 mission profiles varied substantially based on specific research objectives, but were generally classified as either an altitude or a speed profile. The altitude trajectory, such as that of Flight 3-65, consisted of an immediate engine ignition after separation from the NB-52, followed by a $2-$ to $2.5-\mathrm{g}$ pull-up so that the aircraft velocity during the propulsive phase increased from Mach 0.7 at launch to approximately Mach 5. The aircraft then experienced about two minutes of zero-g before a rapid onset of dynamic pressure associated with atmospheric reentry. The aircraft was stabilized at a predetermined entry angle of attack and decelerated at a load of $5 \mathrm{~g}$ before being recovered into an equilibrium glide and set up for landing.

The $\mathrm{X}-15$ program was not without frequent anomalies and a few serious accidents. Aborts due to subsystem malfunctions were commonplace. Owing to the short flight duration, generally poor radio communications, and a high reliance on the research pilot to troubleshoot subsystem issues, the pilot's role in mitigating the effects of component failures was paramount. In nearly all cases where major subsystem anomalies threatened the safety of flight, direct action by the pilot successfully prevented a catastrophic accident [1].

\subsection{THE 1967 X-15-3 CONFIGURATION}

\section{Instrumentation}

Except for supplemental research instrumentation, the instrumentation systems on all the X-15 aircraft used to measure the aircraft dynamic states both for the purposes of flight control and for pilot display were similar. Flight measurement systems included the X15 "ball-nose" aerodynamic flow direction sensor and the Inertial Flight Data System (IFDS), which consisted of an inertial measurement unit (IMU), a dual-redundant rate gyro package, and a computer.

The innovative ball-nose air data system or "Q-ball" on the X-15 was a hypersonic flow direction sensor mounted in the nose of the aircraft [4]. Accurate measurements of angle of attack $(\alpha)$ and sideslip $(\beta)$ at high Mach number could be provided to the pilot down to a dynamic pressure of approximately $50 \mathrm{psf}$. The ball nose was the source of the data for display on the attitude director indicator (ADI) "8-ball," the pilot's primary attitude instrument for dynamic pressures above 50 psf.

The IFDS was used to derive inertial estimates of altitude, altitude rate, and velocity magnitude with acceptable accurate for about 300 seconds of flight [5]. While the IFDS was the primary source of pilot information during the boost phase of the trajectory, it was considered non critical for the remainder of the flight when the availability of more accurate radar velocity data communicated from ground control supplanted the use of inertially derived measurements. 
At 50 psf, the pilot switched the source of attitude data from the Q-ball to IFDS and, at low dynamic pressures, the pilot's Attitude Director Indicator (ADI) display was tied directly to the IMU gimbal resolvers [6]. The $\mathrm{X}-15$ 's ADI was a standard, freely rotating sphere called the " 8 -ball" in the center of the control panel. The ADI was a critical instrument because the typical X-15 flight profile prevented pilots from seeing Earth's horizon until re-entry, so the precision of flight required by the mission and its experiments demanded constant reference to flight instruments.

The ADI in the X-15-3 was significantly different from the displays in the other two X-15 aircraft. During most operations of the X-15 aircraft, the horizontal and vertical needles indicated angle of attack error from preset and sideslip error from zero, respectively. However, the ADI in the X-15-3 aircraft was modified to enable the pilot to switch to an alternate display configuration called the Precision Attitude Indicator (PAI) that was needed to perform certain experiments with the desired precision. In the PAI mode, the horizontal and vertical needles on the ADI indicated fine pitch- and roll-attitude respectively, rather than the usual angle of attack and sideslip.

A separate set of body-fixed gyros, packaged into two identical three-axis assemblies, were used to support the MH-96 AFCS's dual-channel redundant configuration for flight control rate damping [7]. In the event that the gyro output rate exceeded 22.5 degrees/second, the failure monitor was tripped and the input from the gyro to the flight control system was zeroed. Reset was automatic upon reduction of rate below the critical threshold. No indication of this behavior was provided to the pilot [2].

Acceleration measurements from an accelerometer package separate from the IMU were used primarily in the pitch control loop and to provide a load limiting function in the MH-96 [8]. The pilot's display of normal acceleration was provided by a separate, selfcontained accelerometer mounted in the instrument panel [5].

\section{The Flight Control System}

The X-15 flight control system configuration differed based upon the airframe. On all three vehicles, aerodynamic control surfaces consisted of a vertical stabilizer with an all-moving rudder surface and an all-moving horizontal tail with differential actuation for roll control during atmospheric flight [9] (Figure 3.0-1). During exoatmospheric flight when the aerodynamic controls were ineffective, three-axis attitude control was provided by a reaction control system (RCS) consisting of a dual string of monopropellant peroxide jets in the nose and wingtips [10]. Wing trailing surfaces included landing flaps that were deployed only on final approach. Aft speed brakes were available for energy management after engine shutdown. The jettisonable lower ventral fin was used to increase lateral-directional stability above Mach 6, but it was removed from X-15-3 after 1963 when it found it was not needed during high-altitude reentries [1].

The aerodynamic control surfaces were moved by a set of hydraulic actuators supplied by dual-redundant hydraulic systems. The interface to the servocylinders was linked to the pilot inceptors via a set of pushrods and cables with spring bungees and nonlinear gearing for artificial feel [9].

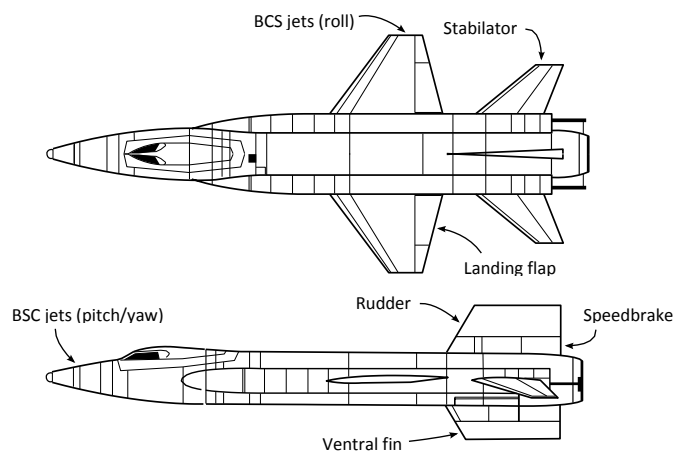

Figure 3.0-1. X-15 Flight Control Effectors

X-15-1 and X-15-2 used a conventional pilot-selectable "fixed-gain" three-axis stability augmentation system SAS with rate gyro feedback having a range of ten preset gains in each axis available for pilot selection during flight. The SAS was required for entry from most flight conditions due to an unstable lateraldirectional mode exhibited by the X-15 at high Mach numbers and moderate angles of attack [11]. Exoatmospheric stabilization relied on the reaction control system (RCS) using either manual inputs (on the left-hand controller) or a rate-damping system, which used one-half (one string) of the reaction control authority in each axis to damp body rates during the ballistic coast [12].

The X-15-3 used a more advanced experimental flight control system, the MH-96, designed to ease the pilot's workload by automatically adjusting the gains of the aircraft's aerodynamic control surfaces in response to changing flight conditions [7,8,13,14]. The MH-96 was referred to as a "self-adaptive" flight control system. A principal function of the MH-96 was to enable automatic blending of the reaction controls and aerodynamic controls during the atmospheric exit and entry transition phases.

The control function of the MH-96 was based on an approach that maintained a small-amplitude limit cycle in the servoactuators of the aerodynamic control surfaces via modulation of the forward gain in the inner loop (Figure 3.0-2). The automated gain changer was designed to maintain the gain of the aerodynamic 
control at its upper stability limit and in doing so was able to maintain a nearly constant aircraft dynamic response over the atmospheric portion of the flight envelope as the dynamic pressure and effectiveness of the aerodynamic control surfaces changed.

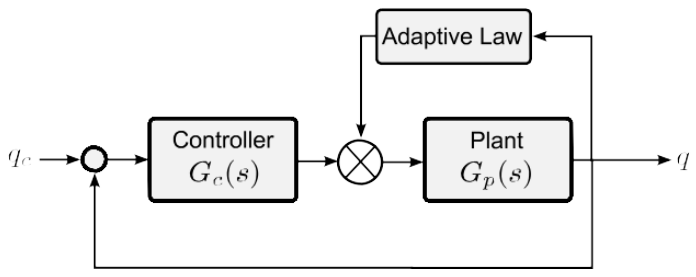

Figure 3.0-2. MH-96 Adaptive Concept

The MH-96 designers recognized that since the adaptive gain was inversely proportional to the control surface effectiveness, the adaptive gain could be used to determine the necessity of supplemental reaction control [7]. To that effect, the MH-96 incorporated an attitude-rate RCS phase plane controller with an "R/C AUTO" mode that automatically engaged and disengaged the reaction control system based upon the value of the sum of the adaptive gains in all three axes. RCS was enabled when the adaptive gains reached $90 \%$ of their maximum sum, and RCS was disengaged at $75 \%$ of the maximum sum [8]. RCS control with the MH-96 was provided through the right-hand control inceptor that was also used for control of the aerodynamic surfaces during atmospheric exit. Manual RCS engage/disengage for the MH-96 as well as the left-hand inceptor was still provided in addition to the "R/C AUTO" mode, but manual control was seldom needed during high-altitude flights with the MH-96.

The gain modulation element of the MH-96 was configured using filtering to respond to changes in only a narrow portion of the frequency spectrum of the sensed control signal, which was taken at the output of the servocylinder. The assumption was that only the small-amplitude limit cycle activity would be present in this narrow frequency range and, thus, would be isolated from other signals below (rigid-body control motions) or above (structural flexibility) this band.

\subsection{FLIGHT 3-65}

Flight 3-65 on November 15, 1967, was planned as an altitude flight. The pilot was conducting his seventh $\mathrm{X}-15$ flight and his third flight in X-15-3.

The two flights immediately preceding Flight 3-65 were also high-altitude flights and were made with the aircraft in nearly the same configuration. The exceptions were that, for purposes of performing additional aerothermal and aerodynamic experiments on Flight 3-65, a panel of ablative material was added to the left-hand upper speed brake to test the adhesive and insulating properties of a material proposed for use on the Saturn launch vehicle, and a traversing probe experiment was added to the starboard wing pod. Most instrumentation and systems, including the traverse probe experiment, were activated just prior to launch.

There were eight primary science objectives, including boost guidance evaluation, solar spectrum and plume measurement experiments, the traversing probe experiment, micrometeorite collection, ablative materials testing, and several instrumentation tests. Orienting the aircraft during the ballistic coast involved use of a precision attitude indicator (PAI) mode of the ADI display to perform a tracking task at or near peak altitude such that the solar spectrum experiment (located in the aft experiment compartment) could be oriented toward the sun.

Following is a summary of Flight 3-65. For a detailed time line, see [3].

Launch from the B-52 took place at 10:30:07 with all systems operating normally, followed by ignition and the power-on phase. At 10:31:28 (just prior to engine shutdown, which occurred at 10:31:30 and at about 140,000 feet altitude), the IFDS computer and instrument malfunction lights illuminated and they could not be reset. At 10:31:33, the pilot switched the ADI to the PAI mode and then initiated the wing-rock maneuver. At 10:31:58, the pilot's report that the computer and instrument malfunction lights were on was acknowledged by ground control. By the time the wing-rock maneuver was completed at 10:32:20, the airplane had started a slow yaw drift to the right, ground control had seen evidence of control problems, and the planned roll angle during the wing-rock maneuver had been exceeded.

Even though ground control knew that the IFDS computer was malfunctioning, the pilot was instructed to perform the computed $\alpha / \beta$-check maneuver, which relied on using the display of IFDS computed angle of attack and computed angle of sideslip. The pilot initiated this maneuver immediately following completion of the wing-rock maneuver. During the maneuver, the aircraft reaction control system did not respond normally to pilot inputs and a negative sideslip gradually increased to off-scale nose right.

At approximately 10:32:50, the pilot initiated the Precision Attitude-Tracking Task in accordance with instructions from ground control. At this time, the sideslip angle had increased to 20 degrees. The aircraft reaction control system momentarily resumed normal operation, arresting the positive yaw rate.

At 10:32:51.2, the Flight Controller reported "Over the top at about 261" [actually, 266 (thousand feet)] and at 10:33:01.4, the Flight Controller told the pilot that he was looking "real good".

However, due to a lack of normal response from the AFCS right-hand reaction control inceptor, at 10:33:05 
the pilot elected to use the left-hand manual control inceptor. By the time the pilot began to use the manual control inceptor, the aircraft had rolled left to zero bank angle and the precision roll indicator needle called for the aircraft to be rolled to the right. However, the pilot applied manual right-yaw reaction control, further increasing the deviation from the planned heading.

At 10:33:39 and about 240,000 feet altitude, the pilot reported that the aircraft control seemed "squirrelly." At 10:34:01, the pilot transmitted, "I'm in a spin." At that time, the aircraft had already reached peak altitude and was in a descent through 210,000 feet. The aircraft attitude was approximately 40 degrees above the horizon and was approximately 90 degrees right of the planned heading with a roll rate of 20 degrees per second. The aircraft had already completed one yaw revolution. The dynamic pressure in this flight condition was still low but increasing through approximately $1.5 \mathrm{psf}$. At this time, the dynamic pressure was still less than $20 \mathrm{psf}$ and reaction controls were required. The pilot reported the spin a second time at 10:34:16.

The aircraft continued uncontrolled motion until about 10:34:34 where, at an altitude of approximately 130,000 feet, the spin was arrested and the aircraft entered into an inverted dive. Three seconds later, the MH-96 AFCS entered into a large-amplitude limit-cycle oscillation (LCO) in the pitch axis with the stabilators moving at their maximum rate of about 26 degrees per second. As dynamic pressure increased during rapid descent, the motions of all the aerodynamic control surfaces produced rapidly increasing accelerations that quickly exceeded structural limits.

At 10:34:54, at an altitude of 62,000 feet and a dynamic pressure exceeding 1300 psf, a catastrophic buckling of the fuselage occurred and the aircraft disintegrated into several large fragments. The pilot did not successfully eject.

\subsection{FAILURE ANALYSIS}

At 10:31:07, when the aircraft reached about 90,000 feet altitude, arcing from the motor drive of the traversing probe caused an electrical disturbance to the aircraft's electrical system that continued until 10:33:53. The electrical disturbance caused failures of the computer in the Inertial Flight Data System (IFDS), of components of the MH-96 AFCS and of electrical components of the aircraft's flight-control system that included spurious electrical signals to the servos that drove the aerosurface actuators. Due to the design of the $\mathrm{MH}-96$, this motion caused random fluctuations in the automated gain control from maximum to minimum. In the following subsections, analyses of individual subsystem failures and their effects will be detailed.

\section{Traverse Probe Experiment Failure}

The traversing probe installed in the starboard-wing pod consisted of a servo-controlled pressure boom whose design was such that it would oscillate across the bow shock boundary, providing a measurement of the location of the shock. The traverse probe relied on power from the aircraft and was interfaced to the primary 115 -volt $400-\mathrm{Hz}$ alternating current (AC) power bus supplied from the aircraft APU generators. The traverse probe experiment had been carried aloft on two prior missions in 1963, although neither achieved an exceptionally high altitude [1]. No anomalies related to the traversing probe were detected on these flights, which may have been due to the relatively short exposure to the high-altitude environment and to the lack of particularly sensitive electronics on the previous flights.

Post-flight tests of the reconstructed traversing probe hardware concluded that below pressures corresponding to approximately 90,000 feet altitude, a starting capacitor associated with the traverse probe drive motor would first develop a corona discharge and then exhibit violent arcing to ground across an approximately 0.25 -inch gap between the capacitor terminal and the chassis of the experiment. Although low-pressure gas dielectric breakdown was already a well-known phenomenon, experiment designers were unaware that the COTS components used in the traverse probe developed voltages that far exceeded the Paschen threshold for arcing at X-15 altitudes.

The electrical isolation for the experiment package did not adequately protect the aircraft main power bus on Flight 3-65 and the effects of the traverse probe's electromagnetic interference were severe [2]. The arcing caused numerous subsystem failures, erratic motion of the servoactuators, fluctuations in the MH-96 AFCS gain changer electronics, and interrupted pilot access to the reaction control system that led to an eventual loss of control.

\section{IFDS Computer Failure}

The Inertial Flight Data System installed on the X-15-1 and X-15-3 aircraft consisted of a digital computer and an analog stable platform (IMU). The IFDS digital computer contained the mechanization of the navigation equations and was used to accumulate velocity data from the stable platform and transform this information into a coordinate frame suitable for use by the pilot and the boost guidance algorithm. The principal outputs of the IFDS were the altitude, altitude rate, and earth-relative velocity magnitude. In addition, body-relative velocity data were used to establish estimates of angle of attack and sideslip. During Flight 3-65, the accuracies of the IFDS-computed angle of attack and sideslip were being experimentally evaluated. 
At the onset of Flight 3-65's electrical disturbance, the IFDS began a series of resets. In each case, transient upsets to the power supply caused the IFDS to temporarily halt integration of the navigation equations and resume shortly thereafter. This event occurred at least 61 times [2] during the less than 3 minutes of electrical disturbance. Due to the loss of acceleration integration during the halt periods, a substantial error accumulated in the IFDS estimates of the altitude, altitude rate, and velocity. Importantly, the failure in the IFDS computer did not affect the ADI display of aircraft attitude; when the ADI display was not being driven by the Q-ball sensor, it was slaved to the IMU resolver angles. Thus, the erroneous data from the IFDS do not appear to be a direct contributor to the vehicle loss of control. However, the devolving state of the IFDS displays, especially in that the altitude and velocity tapes were substantially different from the expected values, combined with repeated warnings from the IFDS error annunciator suggests that the pilot was suspect of any data originating from the IFDS.

The susceptibility of the digital IFDS computer to power supply transients was a known problem, but it had apparently only occurred on X-15-1 and was suspected to have been due to a particularly high electrical load [6].

\section{Servo Transient Anomaly}

Large transients in the servoactuators of the aerodynamic control surfaces during Flight 3-65 were, most likely, due to their sensitivity to the severe electrical noise caused by the traverse probe failure. Some evidence of this sensitivity had been seen on prior flights, but the effects were considered to be benign [2].

Servo transients during Flight 3-65 had two distinct and equally problematic effects. First, the aforementioned coupling with the gain changer electronics caused the MH-96 to interpret the large servo motions as excessive servo limit cycles, and the $\mathrm{MH}-96$ correctly responded by rapidly decreasing the gain to recover stability. In doing so, the logic associated with the automatic RCS blending was activated, and the RCS was disengaged intermittently in synchronization with the servo transients. The pilot was not notified of the disengagement.

Second, the MH-96 AFCS included a failure monitor circuit on each servocylinder to detect hardover failures and completely disengage the stability augmentation for the associated axis (roll, pitch, or yaw) [7]. In the X-15-3's MH-96, detection was triggered when the rate of the servo travel differed in sign from the expected rate. In the event of a "damper reset," two lights were illuminated on the panel to notify the pilot, and pilot action was required to re-engage the failed channels. In order to reduce the potential for supercritical operation in the event that the MH-96 was disengaged at a low dynamic pressure condition and re-engaged at a high dynamic pressure condition, the gain changer electronics were configured to reset the gain to minimum following any reset and delay return to maximum about 10 to 20 seconds. Consequently, in exoatmospheric flight, a reset would interrupt access to the RCS system during that time. During Flight 3-65, this occurred at least twice.

The spurious electrical signals to the aerodynamic servos caused random fluctuations in the automated gain control from maximum to minimum and erratic access to the RCS through the pilot's right side stick. The aircraft had already attained large angles and angular velocities in pitch, roll, and yaw and the aircraft entered into a spin.

\section{Yaw Rate Gyro Channel Disconnect}

In addition to the hardover monitor responsible for the pitch and roll axis disengage, each of two redundant channels of each axis in the MH-96 architecture included a rate hardover monitor on the flight control rate gyro. An ability to recover from a flight control rate gyro fault was a requirement of the MH-96 design, but a simultaneous failure of both gyros in a single axis was not considered credible. Thus, the architecture was designed so a single failure could be accommodated by disconnecting the failed gyro, in which case the total adaptive gain (i.e., the sum of both channels in a given axis) would automatically increase to return the total system gain to a normal range [15]. Unlike the case of a servo failure detection, the adaptive channels were not disengaged, and the pilot was not notified.

The flight envelope provided to the MH-96 designers specified the range of angular rates in each axis would not exceed 20 degrees per second, and the gyro output amplifiers were designed to saturate at 30 degrees per second. As such, the hardover monitor threshold in pitch and yaw was empirically set to a value of approximately 22.5 degrees per second. The roll channel used a differential trigger, but essentially operated the same way.

During Flight 3-65, the onset of a spin brought the true body yaw rate above the hardover failure-disconnect threshold simultaneously in both yaw channels, which were operating normally. As designed, both channels simultaneously disconnected, effectively opening the yaw damper loop and providing no yaw rate feedback from approximately 10 seconds after spin entry until 10:34:29 when the disconnect circuits reset and reenabled both channels.

Lacking any closed-loop feedback, the MH-96 total gain in yaw increased to maximum and remained there so that RCS control was available to the pilot so long 
as the pitch and yaw gains were near maximum. However, regardless of the RCS state, the pilot had transitioned to the left-hand stick and RCS yaw rate damping was unavailable due to the lack of rate gyro inputs. The primary effect of this failure was to exacerbate spin development with no rate damping augmentation.

\section{MH-96 Large-Amplitude Limit Cycle}

Within seconds after the aircraft entered a spin, the MH-96 AFCS went into high-amplitude limit-cycle oscillations of the aerodynamic control surfaces causing load exceedances on the airframe that eventually caused the aircraft to break up at approximately 60,000 feet. The AIB, unfortunately, deemed an analysis of the root causes unnecessary since the only example of the MH-96 had been destroyed [2]. An extensive analysis was recently undertaken by the NASA Engineering and Safety Center (NESC) to isolate the most probable cause of the MH-96 instability and to gain insight into the exact mode(s) of failure [16]. The results are summarized here.

The MH-96 AFCS, coupled to the X-15-3 airframe at a fixed flight condition above approximately Mach 3.0, could exhibit two distinct modes of oscillation. The first was dominated by the desired limit cycle associated with small-amplitude surface motion at the critical forward gain with no rigid body motion. The second was a large-amplitude LCO involving the rigid body and the power actuator but not directly involving the adaptation dynamics. The presence of the LCO on Flight 3-65 appears to have been the result of a latent design oversight in a structural notch filter installed only on the MH-96 configuration.
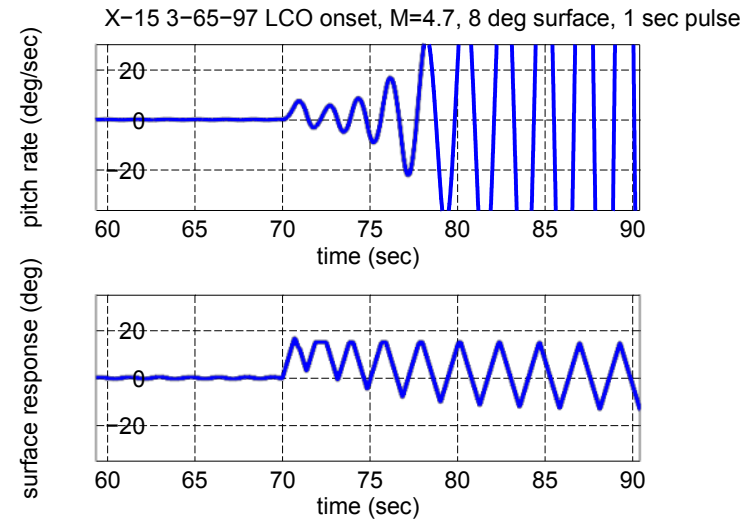

Figure 5.0-1. Flight 3-65 Divergent LCO Reproduced in Nonlinear Simulation ( $M=4.7, q=390 \mathrm{psf})$

Nonlinear time-domain simulation and nonlinear frequency domain (describing function) analysis of a detailed model of the X-15-3 AFCS revealed that transition from the small-amplitude desirable LCO to the large-amplitude destructive LCO was caused by an inner-loop instability due to large system lag in the structural notch filters coupled with the the rate-limited power actuator (Figure 5.0-1). The structural filters differed from the filters used in the fixed-gain SAS on the other aircraft, since the loop shaping requirements were different for the MH-96 adaptive mode. The mechanism by which a destructive limit cycle could be introduced involved an abrupt maneuver at relatively high forward gain, where the power actuator would reach rate saturation while inducing large angular acceleration on the rigid body. If this event occurred at a flight condition where the rigid-body short period damping was particularly low, as it did during Flight $3-65$, the rigid-body oscillation was then divergent, even with fixed adaptive gains. This failure mode is not unlike a classical pilot-induced oscillation (PIO), where the pilot acts as the adaptive gain.

While the adaptation dynamics of the MH-96 AFCS were not directly involved in the LCO phenomenon, the limiting circuitry associated with the MH-96 adaptive loop could be saturated by large DC signals [14]. If this occurred as it did in Flight 3-65, the gains would ramp to maximum in an effort to reestablish the desired high-frequency limit cycle. This behavior further amplified the large-amplitude limit cycle, leading to a loss of rigid-body control.

The MH-96 divergent limit cycle had been briefly encountered once before during an X-15 flight in 1965, but it was considered sufficiently unlikely or benign that steps were not taken to understand and eliminate the behavior [2]. Instead, pilots were cautioned to avoid large inputs at flight conditions that might trigger the LCO.

The investigation following the Flight 3-65 accident concluded from simulation studies that the LCO could have been avoided had the pilot disabled the MH-96 and reverted to fixed-gain operation prior to entry. It is important to emphasize that simply disabling the adaptive mechanism would not have enabled the pilot to recover stability, but since the fixed-gain backup mode also used the lowest acceptable gain setting, the gain was below the threshold that would sustain rate limiting.

The role of the adaptive control law in the divergent limit cycle behavior has been largely misunderstood in the modern flight controls community and has been incorrectly reported in the literature [17]. In the models used for these past studies, the instability was induced by artificially injecting an $80 \%$ asymmetry in stabilator control surface effectiveness (control surface damage), and the modeled actuators, lacking rate limiting, had a bandwidth more than an order of magnitude higher than those of the X-15. 
The analysis of the fatal accident of X-15-3's Flight 3-65 has highlighted several recurring themes in the scenario leading up to and during the flight. As is often the case in accidents, a confluence of latent failure modes and sensitivities was compounded by the unique circumstances of the flight. It is plausible that had one or more of these factors not been present, the accident would not have occurred or the pilot might have recovered control in time to effect a safe landing.

\subsection{Findings}

Insofar as the failures of aircraft systems, the following findings were identified:

- The X-15 3-65 accident was caused by an electrical failure in an experiment package using COTS components that had not been properly qualified for the X-15 flight environment.

- By the time of its deployment, the MH-96 flight control system had undergone multiple modifications to compensate for operational factors not accounted for in its initial concept designs that reduced its overall performance and robustness below that of its theoretically ideal operating characteristics. These changes, such as the installation of a structural notch filter, were not rigorously verified or reconciled with the original design and stability assessment methodology.

- The pilot's inability to recognize and isolate the subsystem failures and safely regain control of the aircraft was largely caused by the lack of adequate display of safety-critical information to the pilot and to ground control.

- $\quad$ Failure detection features of the MH-96 AFCS that were designed to improve reliability, when exercised outside their intended design envelope, malfunctioned and caused degraded performance, which contributed to the loss of control.

- Of the major subsystem failures contributing to the accident, three (i.e., the IFDS computer failure, the servo transient anomaly, and the MH-96 limit cycle) had shown some evidence of failure on previous flights. The program's decision to continue flight operations without assessing risk and identifying the root cause(s) of these anomalies was a causal factor in the 3-65 accident.

\subsection{Recommendations}

The following recommendations follow from the findings of this study:

- The use of high-voltage electronics, and especially AC potentials, in space operations should be minimized to the greatest extent possible. Environmental qualification of high-voltage components for the target environment is mandatory and should include testing at simulated flight conditions, even if they are electrically and mechanically isolated from safety-critical systems.

- The reliability and efficacy of electrical isolation and grounding schemes should be rigorously assessed to ensure that malfunctioning equipment cannot cause cascaded electrical failures.

- The implications and risks of COTS components must be thoroughly assessed by system designers.

- Analysis and design of flight control systems should explicitly account for saturation and rate limiting behavior. Simulations (and when practical, incremental flight testing) should exercise regions outside of the expected flight envelope to include specific cases that induce nonlinear behavior.

- If modifications are made to improve the behavior of a fielded design, those modifications must be reconciled with the baseline design methodology. If the design process is found to be deficient or poorly correlated with flight data, these deficiencies must be resolved and documented.

- An automated system, the failure of which would compromise the safety and/or the operability of the aircraft, must not rely on a single source of aircraft or environmental data for computation of its automated actions.

- A failure of the FCS must be made evident to the pilot immediately. If manual control is possible, a failure must not interfere with the pilot's manual and direct control of the aircraft.

- Flight operations must not continue in the presence of unexplained malfunctions or anomalous behavior in subsystems or components.

\section{REFERENCES}

1. D. Jenkins, "X-15: Extending the Frontiers of Flight,” NASA SP-2007-9-001-HQ, 2007.

2. D. Bellman et al., "Investigation of the Crash of the X-15-3 Aircraft on November 15, 1967," January 1968.

3. Barshi, Immanuel, Orr, Jeb S., and Statler, Irving C., "The Human Factors of an Early Space Accident: Flight 3-65 of the X-15." Proceedings of the $7^{\text {th }}$ Conference of the International Association for the Advancement of Space Safety, Friedrichshafen, Germany, 20-22 October 2014. 
4. J. Cary and E. Keener, "Flight Evaluation of the X-15 Ball-Nose Flow-Direction Sensor as an AirData System," NASA TN D-2923, NASA Flight Research Center, July 1965.

5. J. Fischel and L. Webb, "Flight-Informational Sensors, Display, and Space Control of the X-15 Airplane for Atmospheric and Near-Space Flight Missions," NASA TN D-2407, NASA Flight Research Center, 1964.

6. M. Burke, "X-15 Analog and Digital Inertial Systems Flight Experience," NASA TN D-4642, July 1968.

7. D. Mellen, G. Cole, and J. Lindahl, "Advanced Flight Vehicle Self-Adaptive Flight Control System, Part II: Design of MH-96 System," WADD-TR-60-651, Part II, Flight Control Laboratory, Wright Air Development Division, USAF Air Research and Development Command, February 1961.

8. J. Lindahl, W. McGuire, and M. Reed, "Advanced Flight Vehicle Self-Adaptive Flight Control System, Part V: Acceptance Flight Tests," WADD-TR-60-651, Part V, Flight Control Laboratory, Aeronautical Systems Division, USAF Systems Command, May 1963.

9. R. Tremant, "Operational Experiences and Characteristics of the X-15 Flight Control System," NASA TN D-1402, December 1962.

10. C. Jarvis and E. Adkins, "Operational Experience with X-15 Reaction Controls," NASA TM X-56002, NASA Flight Research Center, April 1964.

11. E. Holleman, "Control Experiences of the X15 Pertinent to Lifting Entry," NASA TN D-3262, NASA Flight Research Center, February 1966.

12. C. Jarvis and W. Lock, "Operational Experience with the X-15 Reaction Control and Reaction Augmentation Systems," NASA TN D-2864, NASA Flight Research Center, June 1965.

13. D. Mellen, "The Development and Flight Test of an Adaptive Flight Control System for the X-15 Vehicle," Technical Report, Minneapolis-Honeywell, February 1963.

14. M. Thompson and J. Welsh, "Flight Test Experience with Adaptive Control Systems,", NASA Flight Research Center, 1970.

15. J. Lindahl, W. McGuire, and M. Reed, "Advanced Flight Vehicle Self-Adaptive Flight Control System, Part VII: Final Report on Study, Development, and Test of the MH-96 System for the X-15," WADD-TR-60-651, Part VII, Air Force Flight Dynamics Laboratory, Research and Technology Division, USAF Systems Command, December 1963.
16. Orr, J., I. Barshi, I. Statler, N. Dennehy, et al., "A Comprehensive Analysis of the X-15 Flight 3-65 Accident," NASA Engineering and Safety Center (NESC) Technical Assessment Report TI-14-008957 (in publication), 2014.

17. Z. Dydek, A. Annaswamy, and E. Lavretsky, "Adaptive Control and the NASA X-15-3 Flight Revisited," IEEE Control Syst. Mag., Vol. 30, No. 3, June 2010, pp. 32-48. 